\title{
Emotions in cognitive conflicts are not aversive but are task specific
}

\author{
Annekathrin Schacht, Olaf Dimigen, ANd Werner Sommer \\ Humboldt-Universität zu Berlin, Berlin, Germany
}

\begin{abstract}
It has been suggested that cognitive conflicts require effortful processing and, therefore, are aversive (Botvinick, 2007). In the present study, we compared conflicts emerging from the inhibition of a predominant response tendency in a go/no-go task with those between incompatible response activations in a Simon task in a within-subjects design, using the same type of stimuli. Whereas no-go trials elicited reduced skin conductance and pupillometric responses, but prolonged corrugator muscle activity, as compared with go trials, incompatible and compatible Simon trials were indistinguishable with respect to these parameters. Furthermore, the conflictsensitive $\mathrm{N} 2$ components of the event-related brain potential were similar in amplitude, but showed significantly different scalp distributions, indicating dissociable neural generator systems. The present findings suggest the involvement of different emotional and cognitive processes in both types of cognitive conflicts - none being aversive, however. In addition, the N2 findings call into question claims of common monitoring systems for all kinds of cognitive conflicts.
\end{abstract}

Cognitive conflicts arise when different and incompatible response tendencies are simultaneously present. For several reasons, cognitive conflicts might be emotionally aversive. In the present study, we assessed the validity of this suggestion and its generalizability across two experimental conflict paradigms.

The assumption that cognitive conflicts are aversive is plausible from different perspectives. Empirically, conflicts seem to be closely linked to performance errors (e.g., Nieuwenhuis, Yeung, van den Wildenberg, \& Ridderinkhof, 2003; Yeung, Botvinick, \& Cohen, 2004), which, in turn, elicit defensive emotional reactions (e.g., Hajcak $\&$ Foti, 2008). One important link between conflicts and performance errors exists in the likely neuroanatomical generators of two prominent components of event-related brain potentials (ERPs): the so-called error-related negativity (ERN or Ne) elicited by performance errors (e.g., Falkenstein, Hohnsbein, Hoormann, \& Blanke, 1991; Gehring, Goss, Coles, Meyer, \& Donchin, 1993) and the $N 2$, which is enhanced in correctly processed incongruent or incompatible conflict trials (e.g., Donkers \& van Boxtel, 2004; van Veen \& Carter, 2002). ERN and N2 show similar distributions over the scalp surface (e.g., van Veen \& Carter, 2002; Yeung et al., 2004) and appear to be generated by the caudal anterior cingulate cortex (ACC), which has been proposed as a gateway for interconnecting emotional and cognitive components of the mind (Bush, Luu, \& Posner, 2000; Devinsky, Morrell, \& Vogt, 1995). On theoretical grounds, Botvinick (2007) recently suggested that conflicts are cognitively demanding and may be registered as aversive because the cognitive system seeks to minimize cognitive effort. Therefore, conflict monitoring may drive a form of avoidance learning in order to bias behavior away from conflict-prone tasks and strategies.

In a series of experiments, Schacht, Nigbur, and Sommer (2009) tested the assumption that conflicts are aversive or at least emotionally arousing by recording peripheral emotion-related indicators in a classical conflict paradigm - the go/no-go task. In go/no-go tasks, there are typically two stimuli, one that has to be responded to (i.e., go stimulus) and one that requires no response (i.e., no-go stimulus). A conflict is said to emerge in no-go trials between the requirement for response inhibition and the preponderant response tendency, especially when no-go trials are relatively infrequent or when "go" responses have high priority (e.g., Band, Ridderinkhof, \& van der Molen, 2003; Nieuwenhuis et al., 2003). Against this theoretical background, we would have expected no-go trials to be more arousing and, specifically, more emotionally aversive than go trials. In contrast, consistently across several experiments, we found no-go trials to be less arousing than go trials, as indicated by smaller skin conductance responses (SCRs). This finding held true for different proportions of go versus no-go stimuli. As to emotional valence, no-go trials were accompanied by reduced startle-blink amplitudes, which are widely accepted as signaling an appetitive rather than an aversive motivational state (Lang, Bradley, \& Cuthbert, 1990). We also found enhanced and prolonged activity of the M. corrugator supercilii in no-go trials. Because the corrugator muscle is activated during negative facial expressions, such as anger, and also by emotionally negative stimuli 
(e.g., Larsen, Norris, \& Cacioppo, 2003), its activity may be seen as indicating an aversive emotional state, contradicting the interpretation for the startle findings. On the other hand, corrugator activation has also been related to situations that are appraised as being obstructive to a given goal (Aue, Flykt, \& Scherer, 2007). With regard to the go/no-go experiments, the preponderant goal would be responding to the go trials; therefore, no-go trials might be seen as being obstructive to this goal. In line with the appraisal account, we concluded from the results of our experiments that no-go trials are neither more arousing nor more aversive than go trials. Instead, they seem to temporarily suspend the prepotent action tendency set up by go trials under the given instructions. Therefore, they might be registered as goal obstructive in the context of a speeded response task. ${ }^{1}$ Before one accepts these findings from go/no-go experiments as a refutation of the idea that conflicts are aversive, it is important to assess whether they generalize to other conflict situations. The presumed conflict between the activation of a preponderant response and its inhibition as required in no-go trials (see Band et al., 2003) might be a special case and might differ from other types of cognitive conflict (such as those between alternative but incompatible responses) with respect to the elicitation of emotions.

In the present study, we compared the effects of conflicts in the go/no-go task with conflicts in the Simon task. The so-called Simon effect (for a review, see Simon, 1990) refers to the fact that spatially arranged responses (e.g., with left or right hands) to nonspatial stimulus features (e.g., stimulus shapes) are faster when the task-irrelevant stimulus location and the response location are compatible than when they are incompatible. According to dualroute models (Kornblum, Hasbroucq, \& Osman, 1990), the Simon effect arises because the spatial position of the stimulus directly activates or primes a response position (direct route), which, for incompatible trials, differs from the response position defined by the instructed stimulusresponse assignment (conditional route). Dual-route models have been embraced by many authors (e.g., Ridderinkhof, 2002), although there are other accounts of the Simon effect as well (cf. Melara, Wang, Vu, \& Proctor, 2008). Electrophysiological studies have provided strong evidence in favor of response conflicts in the Simon tasks by demonstrating early and transient incorrect response activation in the case of incompatible trials followed by activation of the correct response (e.g., Stürmer, Leuthold, Soetens, Schröter, \& Sommer, 2002). Here, we assessed whether, in contrast to no-go trials in the go/no-go task, conflicts between different response activations register as emotionally arousing in incompatible Simon trials.

The need to distinguish among different kinds of conflict on the basis of their emotional effects would be relevant also for the notion of a unitary mechanism of conflict monitoring (e.g., Botvinick, Braver, Barch, Carter, \& Cohen, 2001). The conflict-monitoring theory posits that conflicting response tendencies are monitored in the ACC; a scalp-electric correlate of this activity is the N2 or N200 component in the ERP (Yeung et al., 2004). In line with this suggestion, conflicts in correct trials in the flanker task (e.g., Kopp, Rist, \& Mattler, 1996), in the Stroop task (Liotti, Woldorff, Perez, \& Mayberg, 2000), and in the go/ no-go task (Nieuwenhuis et al., 2003) are accompanied by frontocentral negativities with a source in the ACC (Yeung et al., 2004; Yeung \& Cohen, 2006).

Several studies reported increased N2 components also for incompatible trials in the Simon task: In the time range of the N2, Praamstra and Oostenveld (2003) distinguished a posterior N2pc, ascribed to visuospatial attention, and a more frontal $\mathrm{N} 2 \mathrm{cc}$, originating from bilateral motor cortex associated with visuospatial attentional processes that serve the selection and suppression of competing responses. This functional interpretation seems to be broadly in line with the conflict-monitoring theory. Leuthold and Schröter (2006) found a centrally distributed N2c in an auditory Simon task that was independent of the response modality (hand, eye, or foot) and ascribed it to conflict monitoring or response inhibition. Similarly, Melara et al. (2008) interpreted the function of an N2 component observed in a Simon task as detecting the conflict between the stimulus location and the response location. Thus, current interpretations of $\mathrm{N} 2$ components in Simon tasks are consistent with the conflict-monitoring theory, although other interpretations are possible - for example, in terms of an inhibition of the incorrect response tendency.

Although conflicts in both the go/no-go task and the Simon task elicit negative-going frontocentral components within 200-300 msec, one should bear in mind that other ERP negativities have been described in this latency range as well. These components may reflect the need for or efficiency of response inhibition, action selection, and perceptual mismatching (Folstein \& Van Petten, 2008). Therefore, it is conceivable that, in different conflict tasks, not only does conflict monitoring contribute to negative-going ERPs in the N2 time range, but other cognitive processes contribute as well. Furthermore, the mixture of these components may be task specific. For example, Praamstra and Oostenveld (2003) compared the N2 components in a Simon-type task and a go/no-go task, using the same stimuli, and found clear differences, which indicates that conflict-related N2 effects can differ as a function of task. Although this would not rule out that in each case there is a conflict-monitoring component in the ERP, it would indicate that the relative preponderance of this component among other influences on the N2 or ERN might vary as a function of the particular type of task, even if all tasks may involve conflicts at some level.

In the present study, we directly compared both emotional and conflict-related activity in the go/no-go and the Simon task in a within-subjects design. If the suggestion that conflicts are aversive is correct for the Simon but not for the go/no-go task, we expected emotional arousal of negative valence for incompatible Simon trials, whereas we should replicate our previous findings for no-go trials (Schacht et al., 2009). To test this assumption, we recorded activity from three emotion-related peripheral indicators, namely SCRs, the corrugator, and pupil dilations. Pupil diameter was included as an additional indi- 
cator of emotional arousal (e.g., Bradley, Miccoli, Escrig, \& Lang, 2008; Critchley, Tang, Glaser, Butterworth, \& Dolan, 2005), cognitive processing load, or mental effort, respectively (for reviews, see Andreassi, 2007; Beatty \& Lucero-Wagoner, 2000). Botvinick (2007) suggested that the aversive quality of conflicts is caused by the effort involved in conflict processing. Therefore, the pupillary response in incompatible trials should also be larger because of the increased effort involved in processing the conflicts in this condition.

If Simon-type and go/no-go conflicts indeed differ in terms of affective responses, the claim that both types of conflicts are related to a unitary conflict-monitoring system would deserve a closer look. The similarity of the stimulus conditions in the two tasks employed here allowed for a direct comparison of these monitoring mechanisms as presumably reflected in the N2 component of the ERP.

\section{METHOD}

\section{Participants}

Of the 20 students (10 female, mean age 26.1 years) who participated in the experiment, 16 were right-handed and 4 were left-handed (following Oldfield, 1971). All reported normal or corrected-to-normal vision and no neurological or neuropsychological disorders. Participants gave their informed consent and received $€ 10 / \mathrm{h}$ or course credit.

\section{Stimuli and Procedure}

Participants were seated in a dimly illuminated, sound-attenuated, and electrically shielded chamber, facing a monitor at a distance of $80 \mathrm{~cm}$. All stimuli were presented on a dark gray background. The go/no-go condition replicated our previous experiments in all respects except for the response deadline, which was not used in the present experiment. The capital letters "M" and "W" (Trebuchet MS font) served as imperative stimuli. Trials always started with a white fixation cross, which turned yellow after $900 \mathrm{msec}$. After another $200 \mathrm{msec}$, the fixation cross was replaced by a white letter, presented for $100 \mathrm{msec}$. For the go/no-go task, the letters appeared at the center of the screen; for the Simon task, they were presented $1.07^{\circ}$ to the left or right of the central fixation cross. The next trial started after 900 msec of blank screen. Each task consisted of six experimental blocks of 100 trials each; go and no-go trials and compatible and incompatible Simon trials, respectively, were equiprobable and were randomly ordered within a block.

The order of the two tasks across the first and second halves of an experimental session, the identity of the go stimulus in the go/no-go task ("M" or "W"), the stimulus-response assignment in the Simon task, as well as the change from the stimulus-response assignment from one task to the other were counterbalanced across participants. Therefore, any carryover effects from the go/no-go task to the Simon task and vice versa should have contributed equally to both conditions in a given task.

In the go/no-go task, participants were instructed to respond to one of the letters as quickly as possible with a keypress, whereas no response was required to the other letter. In the Simon task, a speeded choice reaction was required to the letter with the index and middle fingers of the dominant hand. In contrast to our previous go/ no-go experiments (Schacht et al., 2009), no deadline procedure was applied, since it would be rather untypical for the Simon task, but participants were instructed to respond as fast and as accurately as possible. In compatible trials of the Simon task, the spatial position of the stimulus corresponded to the spatial position of the response key (e.g., a "W" requiring a response with the right finger, presented to the right of fixation), whereas in incompatible trials, the spatial positions of the stimulus and the response key did not correspond (e.g., "W" was presented to the left of fixation). For each task, a practice block of 20 trials was given before the experiment proper.

\section{Psychophysiological Recordings and Processing}

SCRs were recorded using a Coulbourn Model S21-22 constantvoltage $(0.5-\mathrm{V})$ skin-conductance coupler. Two $\mathrm{Ag} / \mathrm{AgCl}$ electrodes with $1.0-\mathrm{cm}$ diameters were placed on the thenar and hypothenar eminence of the nondominant palm and filled with ECI electrode gel (Expressive Constructs, Inc., Worcester, MA). Offline, the continuous SCR record was segmented into overlapping epochs of $8,100 \mathrm{msec}$, starting 2,100 msec before the onset of a given (critical) stimulus. Activity of the left M. corrugator supercilii was measured with Sensormedics miniature $\mathrm{Ag} / \mathrm{AgCl}$ electrodes over the left eyebrow (see Fridlund \& Cacioppo, 1986). The raw electromyography (EMG) signal was amplified by a factor of 30,000 at a passband of $8 \mathrm{~Hz}-10 \mathrm{kHz}$ (Coulbourn V76-23a) and was rectified and integrated (Coulbourn V75-04; time constant $=0.1 \mathrm{sec}$ ). Offline, a lowpass filter of $100 \mathrm{~Hz}$ was applied, and 800-msec epochs, starting $200 \mathrm{msec}$ before stimulus onset, were analyzed. Epochs containing eyeblinks were discarded, on the basis of the vertical electrooculogram (see below). EMG in artifact-free trials was referred to a 200-msec prestimulus baseline and normalized by $z$ transformations within the whole interval from 0 to $600 \mathrm{msec}$ for each participant. Pupillometric responses were recorded with a table-mounted infrared video-based eyetracker (IViewX Hi-Speed 1250 system) at a $500-\mathrm{Hz}$ sampling rate (cf. Dimigen, Valsecchi, Sommer, \& Kliegl, 2009). Head movements were limited by the eyetracker's built-in chin and forehead rest. Horizontal and vertical pupil diameter was recorded from the right eye, but viewing was binocular. To obtain absolute pupil diameters, the system was calibrated before each session with an artificial pupil of known diameter that was attached to the participant's closed eyelid. Offline, pupil data were filtered with a 10-Hz low-pass filter (zero-phase) and cut into 3.5-sec segments, starting $1,000 \mathrm{msec}$ before stimulus onset. Segments were baselinecorrected with a $200-\mathrm{msec}$ prestimulus baseline; those containing eyeblinks or for which data were missing were discarded.

ERPs were recorded from $34 \mathrm{Ag} / \mathrm{AgCl}$ electrodes located according to the extended 10-20 system (Pivik et al., 1993) and referenced to the left mastoid. Most electrodes were placed in an electrode cap (EasyCap). External electrodes were used for the vertical and horizontal electrooculogram, and left and right mastoid. Forehead electrodes Fp1 and Fp2 were foam cushioned to preclude pressure artifacts from contact with the eyetracker's forehead rest. Electrode impedance was kept below $5 \mathrm{k} \Omega$, using ECI electrode gel. All channels were amplified with a passband of $0.05-70 \mathrm{~Hz}$; sampling rate was $250 \mathrm{~Hz}$. Offline, the continuous electroencephalogram record was filtered with a $30-\mathrm{Hz}$ low-pass filter, converted to average reference, and segmented into epochs of 1,200 msec, starting $200 \mathrm{msec}$ before stimulus onset. Epochs containing artifacts were automatically discarded when any amplitude exceeded -100 or $+100 \mu \mathrm{V}$ or when any voltage step exceeded $50 \mu \mathrm{V}$ per sampling point in any of the channels. Average ERPs were generated for each participant, electrode, and experimental condition. All ERPs were referred to a 100 -msec prestimulus baseline.

\section{Data Analysis}

For all dependent variables (ERP, SCR, corrugator activity, and pupillary response), only trials with correct responses were analyzed in separate repeated measures ANOVAs for each task, involving the factor conflict (go vs. no-go and compatible vs. incompatible, respectively). Analogous to our previous study, mean SCR amplitudes were assessed between 2,000 and 3,500 msec. The feasibility of using SCR amplitude measures at short interstimulus intervals (ISIs) in go/no-go tasks was shown by Recio, Schacht, and Sommer (2009), who varied the ISI from 2 to 5 to $8 \mathrm{sec}$. In all ISIs, SCR amplitudes showed a local maximum between 2 and $3.5 \mathrm{sec}$. The SCR amplitudes measured during this interval were consistently smaller 
in the no-go than in the go conditions, irrespective of the ISI. We are therefore confident that the short ISI used in the present study and in our previous study (Schacht et al., 2009) does not invalidate the SCR amplitude measurements.

Mean corrugator activity was calculated between 300 and $600 \mathrm{msec}$ after stimulus onset. Pupil dilations were obtained as mean horizontal diameter between 1,000 and 1,500 msec. ${ }^{2}$ In ANOVAs of ERP data, an additional factor, electrode site, was included. Interactions between an experimental factor and electrode in these ANOVAs may reflect either differences in overall ERP activity (amplitude) or differences in the scalp distributions between experimental conditions. To assess whether the effects obtained in the ANOVAs within a given task were distinguishable with regard to their scalp distributions, overall amplitude differences were eliminated by using normalization (profile analyses; McCarthy \& Wood, 1985). In contrast, global field power (GFP; Lehmann \& Skrandies, 1980) reflects the overall ERP activity across the scalp at any given moment - that is, the root-mean square of voltages at all electrodes - and indicates differences in overall amplitude of the ERP signal.

\section{RESULTS}

\section{Performance}

Mean RT for go trials in the go/no-go task was $372 \mathrm{msec}$. False alarm rates in no-go trials and missing responses in go trials were low $(1.5 \%$ and $2.5 \%$, respectively) and thus were not analyzed further. Typical for the Simon task, RTs and error rates for incompatible trials (means, $511 \mathrm{msec}$ and $7.1 \%$ ) were significantly larger $[F(1,19)=44.2, p<.001$, and $F(1,19)=8.9, p<.01]$, as compared with compatible trials (means, $482 \mathrm{msec}$ and $3.2 \%)$.

\section{Peripheral Emotion Indicators}

As can be seen in Figure 1A and Table 1, we replicated our previous findings in the go/no-go task, since corrugator activity was significantly enhanced to no-go as compared with go trials $\left[F(1,19)=4.8, p<.05, \eta^{2}=\right.$ .200]. Also in line with our previous results, no-go trials seemed to be less arousing than go trials, as reflected in reduced SCRs $\left[F(1,19)=5.8, p<.05, \eta^{2}=.233\right]$ as well as in smaller pupil dilations $[F(1,16)=34.8, p<.001$, $\left.\eta^{2}=.699\right]$. In contrast, none of the peripheral emotion indicators showed any differences between incompatible and compatible trials in the Simon task, neither SCRs and pupil responses $\left[F \mathrm{~s}<1, \eta^{2}=.036\right.$ and .005$]$ nor corrugator activity $\left[F(1,19)=2.1, p>.05, \eta^{2}=.096\right]$.
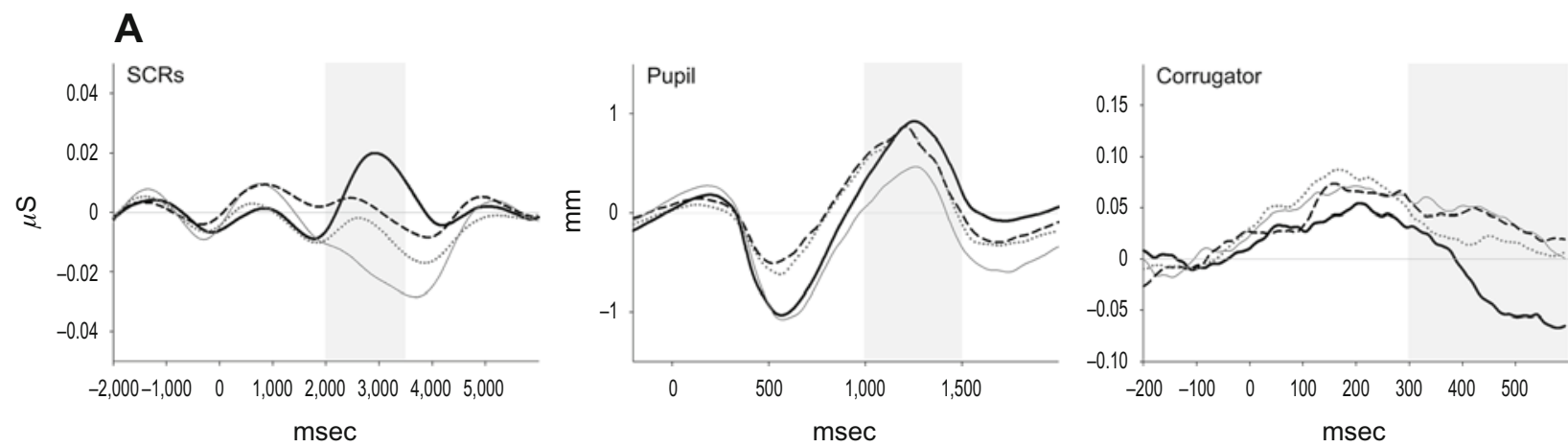

B
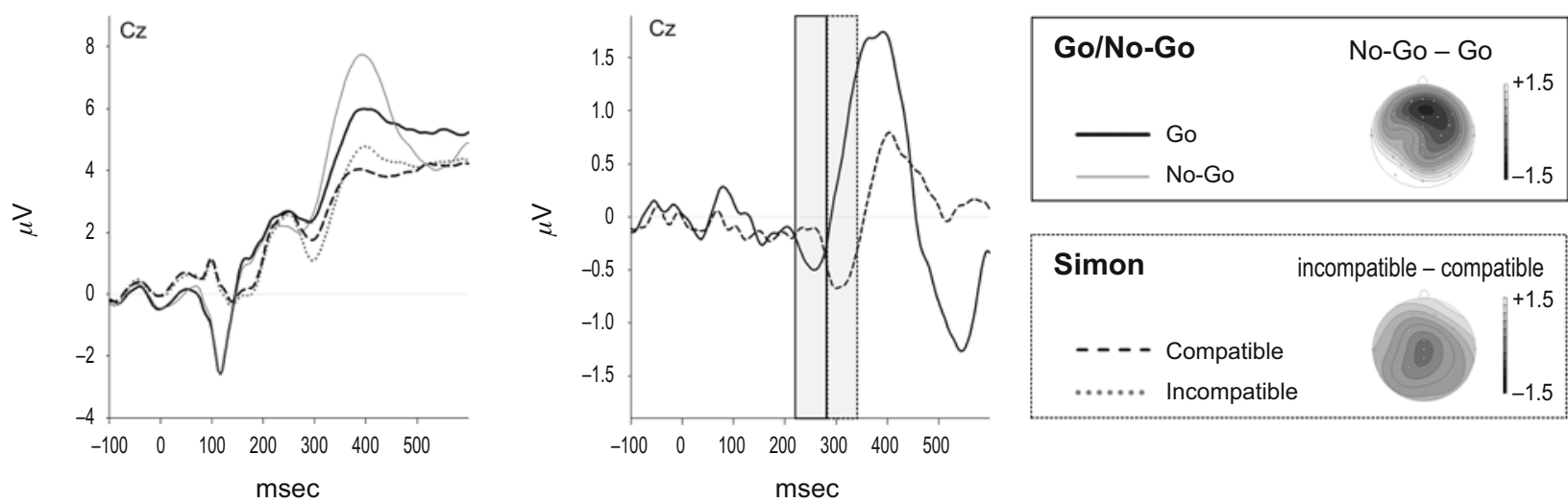

Figure 1. Emotion- and conflict-related indicators in response to go/no-go and compatible and incompatible Simon trials. (A) Grand averages for SCRs (left), pupil size (center), and corrugator activity (right) are contrasted for go, no-go, compatible, and incompatible Simon trials. Gray shading indicates the time intervals used for statistical analyses. Pupillometric responses are plotted as change in pupil diameter relative to the prestimulus baseline interval. (B) The left panel shows grand average ERPs at the Cz electrode in all experimental conditions. In the middle panel, ERP difference waves between no-go and go (solid line) and incompatible and compatible Simon (dashed line) trials are shown. Light gray boxes mark time intervals in which the N2 component appeared to be most pronounced in both conditions. As depicted by the heads in the right panel, the scalp distribution of the no-go N2 has a more anterior maximum as compared with the conflict $\mathbf{N} 2$ in Simon trials. 
Table 1

Descriptive Statistics of Peripheral Indicators in Both Go/No-Go and Simon Tasks

\begin{tabular}{|c|c|c|c|c|c|c|}
\hline \multirow[b]{2}{*}{ Task } & \multicolumn{2}{|c|}{ SCRs $(\mu \mathrm{S})$} & \multicolumn{2}{|c|}{$\begin{array}{c}\text { Pupil } \\
\text { Dilations (mm) }\end{array}$} & \multicolumn{2}{|c|}{$\begin{array}{c}\text { Corrugator } \\
\text { (z Values) }\end{array}$} \\
\hline & $M$ & $S D$ & $M$ & $S D$ & $M$ & $S D$ \\
\hline \multicolumn{7}{|l|}{ Go/No-Go Task } \\
\hline Go & 0.01 & 0.03 & 0.74 & 0.40 & -.03 & .14 \\
\hline No-go & -0.02 & 0.04 & 0.19 & 0.33 & .03 & .13 \\
\hline \multicolumn{7}{|l|}{ Simon Task } \\
\hline Compatible & 0.01 & 0.05 & 0.62 & 0.34 & .04 & .15 \\
\hline Incompatible & 0.00 & 0.04 & 0.66 & 0.40 & .02 & .16 \\
\hline
\end{tabular}

\section{ERPs}

As shown in Figure 1B, conflict trials in both tasks (no-go and incompatible Simon trials) elicited enhanced amplitudes in the N2 time range. Overall, ANOVAs revealed significant differences between ERPs to no-go as compared with go trials between 220 and $280 \mathrm{msec}$ $\left[F(33,627)=9.1, p<.001, \varepsilon=.133, \eta^{2}=.325\right]$ and to incompatible as compared with compatible Simon trials between 280 and $330 \mathrm{msec}[F(33,627)=3.9, p<.01$, $\left.\varepsilon=.168, \eta^{2}=.170\right]$. Interestingly, post hoc analyses revealed that this effect of task was not due to amplitude differences, as indicated by an ANOVA on GFP measures $\left[F(1,19)=2.0, p=.169, \eta^{2}=.094\right]$. Instead, and as becomes obvious from Figure 1B, the N2 components differed in scalp distribution, since the maximum of the N2 to incompatible relative to compatible Simon trials was located more posterior than the maximum of the N2 to no-go as compared with go trials. This impression was verified by an additional ANOVA conducted on normalized difference waves $[F(33,627)=8.4, p<.001, \varepsilon=$ $.169, \eta^{2}=.307$; see McCarthy \& Wood, 1985].

\section{DISCUSSION}

With the present study, we aimed to provide direct evidence for the recent proposal that cognitive conflicts are aversive events as has been suggested on empirical and theoretical grounds (e.g., Botvinick, 2007). Following these suggestions, one should expect changes in peripheral indicators, which have been shown to be sensitive to emotional processing in several previous studies (e.g., Beatty \& Lucero-Wagoner, 2000; Bradley, Codispoti, Cuthbert, \& Lang, 2001). However, results are inconsistent with the suggestion that conflicts are aversive or at least arousing. First, replicating our previous findings (Schacht et al., 2009), we found reduced SCRs and pupil dilations in no-go trials, indicating reduced sympathetic activation to no-go stimuli. At least for the SCRs, this is difficult to attribute to demands on motor exertion (Pugh, Oldroyd, Ray, \& Clark, 1966). The relatively enhanced corrugator activity in no-go trials is also in line with our previous findings and may be interpreted as a consequence of the goal obstructiveness of no-go trials. Although there was no emphasis on fast responses in the present study, results were very similar to our previous findings, indicating that time pressure is not essential for the diminished autonomic responses to no-go trials. It might be noteworthy that several theoretical conceptions of emotions claim a relationship between negative valence and enhanced arousal (e.g., Lang, Bradley, \& Cuthbert, 1997; Russell, 1980), an assumption that is supported by empirical evidence (e.g., Bradley et al., 2001). The diminished autonomic activation to no-go trials therefore already speaks against the idea of conflict's aversiveness in the case of no-go trials. Together, the present findings replicate and extend our previous findings that no-go conflicts are not aversive and less arousing than go trials. This supports our hypothesis that no-go trials may provide temporary relief and register as being relaxing and pleasant rather than aversive (Schacht et al., 2009).

Second, none of the recorded peripheral indicators showed any differences between compatible and incompatible trials in the Simon task, indicating that conflict trials in this task are just as arousing and goal conducive as nonconflict trials. A similar insensitivity of a peripheral indicator to conflict trials has been demonstrated for the pupillary response in the Stroop task by Critchley et al. (2005), whereas a similarly diminished response in no-go as compared with go trials has previously been reported by Richer, Silverman, and Beatty (1983).

The results in the go/no-go paradigm are therefore difficult to reconcile with the notion that all cognitive conflicts are aversive or, at least, arousing. Moreover, they call into question that cognitive conflicts are aversive at all, at least for the two conflict tasks used here. At an even more basic level, the absence of a compatibility effect on the pupil in the Simon task is at variance with the assumption that conflicts are mentally effortful (Botvinick, 2007). The pupillary response is a sensitive indicator for the cognitive demands imposed by a task, as demonstrated by difficulty manipulations in many different experimental tasks (for reviews, see Andreassi, 2007; Beatty \& Lucero-Wagoner, 2000). Therefore, the absence of a compatibility effect in the pupil diameter is difficult to reconcile with the claim that conflicts are more effortful in a general sense. Possibly, the undisputed increased temporal demands in processing incompatible trials are unrelated to those cognitive demands reflected in the pupil diameter. Of course, more research is needed to test this idea in other conflict tasks; nevertheless, our null results from the Simon task and the diminished pupillary response in no-go trials are at variance with the idea that processing of all cognitive conflicts requires more mental effort than do conflict-free conditions.

It seems difficult to argue that our null results in the Simon task relate to any insensitivity of our procedure or our indicators. The very same stimulation procedure that had shown repeated and exquisite sensitivity to autonomic and skeletomuscular changes in the go/no-go task was unable to uncover such effects in the Simon task in a repeated measures design. For the SCR and corrugator activity, the effect sizes were small to medium and medium to large, respectively, but they were in the direction opposite the one predicted according to the assumption 
that conflicts are aversive (cf. Figure 1A). For the pupil diameter, effect size was very small $(<.1)$. It is therefore unlikely that the null results for the Simon task reflect a lack of experimental power. In addition, the failure to detect emotional activations in the Simon task cannot be related to any peculiarities of our procedure, because the Simon paradigm used here yielded the expected effects in every respect, including sequential adaptations in performance. ${ }^{3}$

Our findings also contradict the theoretical explanation for a set of results recently provided by van Steenbergen, Band, and Hommel (2009), who showed an influence of rewarding events on conflict adaptation in the flanker task; that is, the postconflict adaptation was reduced after reward. Van Steenbergen et al. explained their findings by assuming that the reward had counteracted the aversiveness of the conflicts and, thus, reduced the need for adaptive strategies. Whether flanker conflicts may indeed be aversive remains to be demonstrated. Instead, one may suggest that the findings of van Steenbergen et al. are in line with other evidence that positive affect, as induced by reward, may enhance cognitive fluency and modulate executive functions in the favor of adaptive behavior (e.g., Dreisbach \& Goschke, 2004; for a review, see Mitchell \& Phillips, 2007). The effects may exist without the need for any particular affect induced by the experimental taskthat is, by the conflict trials themselves.

Since the present data cast doubt on the suggestion of a global learning signal based on the aversiveness of conflicts, another assumption about a conflict-general mechanism, namely conflict monitoring, is of interest. If the emotions induced by cognitive conflicts are specific rather than global, as shown here for no-go and Simon conflicts, it may relate to the specificity, rather than the generality, of a purported underlying conflict-monitoring mechanism in the brain. As is outlined above, the N2 component in the ERP has been taken as an index of a global conflict-monitoring mechanism. In the present study, we assessed the paradigm specificity of the N2 component by directly comparing ERP responses to conflict (no-go, incompatible) as compared with nonconflict trials (go, compatible Simon trials). Importantly, the N2 component to no-go trials showed a frontocentral maximum, in line with previous results (e.g., Nieuwenhuis et al., 2003). In contrast, the N2 component to incompatible Simon trials showed a negativity over parietocentral electrode sites. These conflict-specific differences in scalp topography, confirmed by significant differences in the profile analysis, indicate that the brain processes underlying the N2 differed in some respect. The present findings are, therefore, in line with reports by Praamstra and Oostenveld (2003) and Nigbur and Stürmer (2008) of distinct N2 distributions in go/no-go and Simon tasks.

Although it is difficult to tell exactly what determines the differences in the conflict-related negativities in the go/no-go task and the Simon task, it is likely that spatial attention is required to resolve the conflict between the spatial position of the stimulus and the spatial position of the selected response in incompatible Simon trials. This employment of spatial attention is known to elicit a posterior N2pc component (Praamstra \& Oostenveld, 2003; cf. Folstein \& Van Petten, 2008). In no-go trials, no such spatial attention is necessary. Instead, the inhibition of the prepotent response may dominate (Falkenstein, Hoormann, \& Hohnsbein, 1999). Regardless of whether there is also a conflict-monitoring component, the different mixtures of subprocesses and their electrophysiological manifestations would cause differences in scalp topographies of the conflict-related negativities. In any case, the present findings indicate the need for future research in search of a task-independent electrophysiological signature of conflict monitoring.

Given the close relationship between error processing and affect, as well as the purported relationship between error processing and conflict processing, why is there no relationship between conflict processing and affect? What, if any, conditions might render conflicts emotional? According to appraisal theories, emotions are elicited when events are of relevance for the person (e.g., Scherer, 2001). It is possible that, under many circumstances, conflict trials in experimental tasks are of no different relevance for the participant's goals than are nonconflict trials. We therefore suggest that conflicts elicit emotional responses only if they differ from nonconflict conditions in a personally relevant way. For example, conflicts should elicit emotions if they endanger the receipt of reward or increase the likelihood of punishment. In most experimental set-ups, conflict trials are no different from no-conflict trials in terms of subjectively accessible consequences as long as the correct response can be executed. This may be different for errors, in which processing has failed and requires adjustments (i.e., some action on the part of the operator), a case for which appraisal theories would predict emotional effects.

The present study shows that conflicts are not necessarily aversive and may neither elicit emotional responses nor require effortful processing. These findings pose a problem to recent suggestions that the aversive emotional quality of conflicts may provide a learning signal for avoiding mentally effortful situations, at least in a general sense. We also provided further evidence that the specificity of conflicts in different tasks may extend also to the monitoring systems of the brain, which may be either conflict specific or superimposed with additional conflict-specific processes and their corresponding ERP components.

\section{AUTHOR NOTE}

We thank Nele Adler and Mareike Bayer for assisting in data collection and analyses, and Rainer Kniesche and Thomas Pinkpank for technical support. This research was supported by the German Research Foundation, Grant So 177/17-1 to W.S. Correspondence concerning this article should be addressed to A. Schacht, Department of Psychology, Humboldt-Universität zu Berlin, Unter den Linden 6, D-10099 Berlin, Germany (e-mail: schachta@hu-berlin.de).

\section{REFERENCES}

ANDREASsi, J. L. (2007). Psychophysiology: Human behavior and physiological response (5th ed.). Mahwah, NJ: Erlbaum.

Aue, T., Flykt, A., \& Scherer, K. R. (2007). First evidence for dif- 
ferential and sequential efferent effects of stimulus relevance and goal conduciveness appraisal. Biological Psychology, 74, 347-357. doi:10.1016/j.biopsycho.2006.09.001

BAND, G. P. H., RidDERINKHOF, K. R., \& VAN DER Molen, M. W. (2003). Speed-accuracy modulation in case of conflict: The roles of activation and inhibition. Psychological Research, 67, 266-279.

Beatty, J., \& Lucero-Wagoner, B. (2000). The pupillary system. In J. T. Cacioppo, L. G. Tassinary, \& G. G. Berntson (Eds.), Handbook of psychophysiology (2nd ed., pp. 142-162). Cambridge: Cambridge University Press.

BotvinicK, M. M. (2007). Conflict monitoring and decision making: Reconciling two perspectives on anterior cingulate function. Cognitive, Affective, \& Behavioral Neuroscience, 7, 356-366. doi:10.3758/ CABN.7.4.356

Botvinick, M. M., Braver, T. S., Barch, D. M., Carter, C. S., \& Cohen, J. D. (2001). Conflict monitoring and cognitive control. Psychological Review, 108, 624-652.

Bradley, M. M., Codispoti, M., Cuthbert, B. N., \& Lang, P. J. (2001). Emotion and motivation I: Defensive and appetitive reactions in picture processing. Emotion, 1, 276-298.

Bradley, M. M., Miccoli, L., Escrig, M. A., \& Lang, P. J. (2008). The pupil as a measure of emotional arousal and autonomic activation. Psychophysiology, 45, 602-607. doi:10.1111/j.1469-8986 .2008.00654.x

Bush, G., LuU, P., \& Posner, M. I. (2000). Cognitive and emotional influences in anterior cingulate cortex. Trends in Cognitive Sciences, 4, 215-222.

Critchley, H. D., Tang, J., Glaser, D., Butterworth, B., \& Dolan, R. J. (2005). Anterior cingulate activity during error and autonomic response. Neurolmage, 27, 885-895.

Devinsky, O., Morrell, M. J., \& Vogt, B. A. (1995). Contributions of anterior cingulate cortex to behaviour. Brain, 118, 279-306.

Dimigen, O., Valsecchi, M., Sommer, W., \& Kliegl, R. (2009). Human microsaccade-related visual brain responses. Journal of Neuroscience, 29, 12321-12331. doi:10.1523/JNEUROSCI.0911-09.2009

Donkers, F. C. L., \& van Boxtel, G. J. M. (2004). The N2 in go/no-go tasks reflects conflict monitoring not response inhibition. Brain \& Cognition, 56, 165-176.

Dreisbach, G., \& GoschKe, T. (2004). How positive affect modulates cognitive control: Reduced perseveration at the cost of increased distractibility. Journal of Experimental Psychology: Learning, Memory, \& Cognition, 30, 343-353.

Falkenstein, M., Hohnsbein, J., Hoormann, J., \& Blanke, L. (1991). Effects of crossmodal divided attention on late ERP components: II. Error processing in choice reaction tasks. Electroencephalography \& Clinical Neurophysiology, 78, 447-455.

Falkenstein, M., Hoormann, J., \& Hohnsbein, J. (1999). ERP components in Go/Nogo tasks and their relation to inhibition. Acta Psychologica, 101, 267-291.

Folstein, J. R., \& Van Petten, C. (2008). Influence of cognitive control and mismatch on the N2 component of the ERP: A review. Psychophysiology, 45, 152-170.

Fridlund, A. J., \& CACIOPPO, J. T. (1986). Guidelines for human electromyographic research. Psychophysiology, 23, 567-589. doi:10.1111/j.1469-8986.1986.tb00676.x

Gehring, W. J., Goss, B., Coles, M. G. H., Meyer, D. E., \& DonCHIN, E. (1993). A neural system for error detection and compensation. Psychological Science, 4, 385-390.

HAJCAK, G., \& FOTI, D. (2008). Errors are aversive: Defensive motivation and the error-related negativity. Psychological Science, 19, 103108

Kopp, B., Rist, F., \& Mattler, U. (1996). N200 in the flanker task as a neurobehavioral tool for investigating executive control. Psychophysiology, 33, 282-294.

Kornblum, S., Hasbroucq, T., \& Osman, A. (1990). Dimensional overlap: Cognitive basis for stimulus-response compatibility-A model and taxonomy. Psychological Review, 97, 253-270.

Lang, P. J., Bradley, M. M., \& Cuthbert, B. N. (1990). Emotion, attention, and the startle reflex. Psychological Review, 97, 377-395. doi:10.1037/0033-295X.97.3.377

Lang, P. J., Bradley, M. M., \& Cuthbert, B. N. (1997). Motivated attention: Affect, activation, and action. In P. J. Lang, R. F. Simons, \&
M. T. Balaban (Eds.), Attention and orienting: Sensory and motivational processes (pp. 97-134). Mahwah, NJ: Erlbaum.

Larsen, J. T., Norris, C. J., \& CACioppo, J. T. (2003). Effects of positive and negative affect on electromyographic activity over zygomaticus major and corrugator supercilii. Psychophysiology, 40, 776-785.

Lehmann, D., \& Skrandies, W. (1980). Reference-free identification of components of checkerboard-evoked multichannel potential fields. Electroencephalography \& Clinical Neurophysiology, 48, 609-621.

Leuthold, H., \& Schröter, H. (2006). Electrophysiological evidence for response priming and conflict regulation in the auditory Simon task. Brain Research, 1097, 167-180.

Liotti, M., Woldorf, M. G., Perez, R., III, \& Mayberg, H. S. (2000). An ERP study of the temporal course of the Stroop color-word interference effect. Neuropsychologia, 38, 701-711.

McCarthy, G., \& Wood, C. C. (1985). Scalp distributions of eventrelated potentials: An ambiguity associated with analysis of variance models. Electroencephalography \& Clinical Neurophysiology, 62, 203-208

Melara, R. D., Wang, H., Vu, K.-P. L., \& Proctor, R. W. (2008). Attentional origins of the Simon effect: Behavioral and electrophysiological evidence. Brain Research, 1215, 147-159.

Mitchell, R. L. C., \& Phillips, L. H. (2007). The psychological, neurochemical and functional neuroanatomical mediators of the effects of positive and negative mood on executive functions. Neuropsychologia, 45, 617-629.

Nieuwenhuis, S., Yeung, N., van den Wildenberg, W., \& RidDERINKHOF, K. R. (2003). Electrophysiological correlates of anterior cingulate function in a go/no-go task: Effects of response conflict and trial type frequency. Cognitive, Affective, \& Behavioral Neuroscience, 3, 17-26.

NigbUR, R., \& StÜRMER, B. (2008). Dissociable conflict adaptation processes in a combined Go/NoGo paradigm [Abstract]. In W. Gevers, B. Liefooghe, \& W. Notebaert (Eds.), Neuroscience and Cognitive Control: Program and abstracts (p. 13). Ghent, Belgium: Ghent University, Experimental Psychology Department.

OldFIELD, R. C. (1971). The assessment and analysis of handedness: The Edinburgh inventory. Neuropsychologia, 9, 97-113.

Pivik, R. T., Broughton, R. J., Coppola, R., Davidson, R. J., Fox, N., \& NuwER, M. R. (1993). Guidelines for the recording and quantitative analysis of electroencephalographic activity in research contexts. Psychophysiology, 30, 547-558.

Praamstra, P., \& Oostenveld, R. (2003). Attention and movementrelated motor cortex activation: A high-density EEG study of spatial stimulus-response compatibility. Cognitive Brain Research, 16, 309322.

Pugh, L. A., Oldroyd, C. R., Ray, T. S., \& Clark, M. L. (1966). Muscular effort and electrodermal responses. Journal of Experimental Psychology, 71, 241-248. doi:10.1037/h0022834

Recio, G., Schacht, A., \& Sommer, W. (2009). Effect of inter-stimulus interval variation on skin conductance responses and event-related potentials in a Go/NoGo task. Biological Psychology, 80, 246-250. doi:10.1016/j.biopsycho.2008.10.007

Richer, F., Silverman, C., \& Beatty, J. (1983). Response selection and initiation in speeded reactions: A pupillometric analysis. Journal of Experimental Psychology: Human Perception \& Performance, 9, 360-370. doi:10.1037/0096-1523.9.3.360

RIDDERINKHOF, K. R. (2002). Activation and suppression in conflict tasks: Empirical clarification through distributional analyses. In W. Prinz \& B. Hommel (Eds.), Common mechanisms in perception and action: Attention and performance XIX (pp. 494-519). Oxford: Oxford University Press.

Russell, J. A. (1980). A circumplex model of affect. Journal of Personality \& Social Psychology, 39, 1161-1178.

Schacht, A., Nigbur, R., \& Sommer, W. (2009). Emotions in Go/ NoGo conflicts. Psychological Research, 73, 843-856.

Scherer, K. R. (2001). Appraisal considered as a process of multilevel sequential checking. In K. R. Scherer, A. Schorr, \& T. Johnstone (Eds.), Appraisal processes in emotion: Theory, methods, research (pp. 92-120). Oxford: Oxford University Press.

SimON, J. R. (1990). The effects of an irrelevant directional cue on human information processing. In R. W. Proctor \& T. G. Reeve (Eds.), 
Stimulus-response compatibility: An integrated perspective (pp. 3186). Amsterdam: Elsevier.

Stürmer, B., Leuthold, H., Soetens, E., Schröter, H., \& SomMER, W. (2002). Control over location-based response activation in the Simon task: Behavioral and electrophysiological evidence. Journal of Experimental Psychology: Human Perception \& Performance, 28, 1345-1363.

van Steenbergen, H., Band, G. P. H., \& Hommel, B. (2009). Reward counteracts conflict adaptation: Evidence for a role of affect in executive control. Psychological Science, 20, 1473-1477. doi:10.1111/j.1467-9280.2009.02470.x

VAN VEen, V., \& CARTER, C. S. (2002). The timing of action-monitoring processes in the anterior cingulate cortex. Journal of Cognitive Neuroscience, 14, 593-602.

Yeung, N., Botvinick, M. M., \& Cohen, J. D. (2004). The neural basis of error detection: Conflict monitoring and the error-related negativity. Psychological Review, 111, 931-959.

Yeung, N., \& Cohen, J. D. (2006). The impact of cognitive deficits on conflict monitoring: Predictable dissociations between the errorrelated negativity and N2. Psychological Science, 17, 164-171.

\section{NOTES}

1. Note that goal obstructiveness is not always aversive, as one might assume. For example, if the goal is performing an unpleasant action, such as correcting exams, signals that interrupt the action, such as incoming e-mails, may be goal obstructive, but, at the same time, pleasant rather than aversive.

2. We excluded data of 3 participants from analyses of pupillometric responses, because the pupil was not properly tracked.

3. An additional analysis on RTs revealed the typical pattern of conflict-adaptation effects, with shortest RTs for compatible-followingcompatible trials $(M=458 \mathrm{msec})$ and-compared with this condition-prolonged RTs to compatible-following-incompatible trials $(M=506 \mathrm{msec})$ and to incompatible-following-compatible trials $(M=$ $526 \mathrm{msec}$; mean for incompatible-following-incompatible trials = $496 \mathrm{msec})$.

(Manuscript received August 17, 2009; revision accepted for publication February 21, 2010.) 